medRxiv preprint doi: https://doi.org/10.1101/2020.10.30.20223529; this version posted November 4, 2020. The copyright holder for this preprint (which was not certified by peer review) is the author/funder, who has granted medRxiv a license to display the preprint in perpetuity

It is made available under a CC-BY-NC-ND 4.0 International license.

\title{
Mapping the human helminthiases: advances and gaps in neglected disease surveillance
}

Catherine G. Schluth ${ }^{1}$, Claire J. Standley², Shweta Bansal ${ }^{1}$ and Colin J. Carlson ${ }^{1,2, \dagger}$

${ }^{1}$ Department of Biology, Georgetown University, Washington, D.C. 2005\%, USA. ${ }^{2}$ Center for Global Health Science and Security, Georgetown University Medical Center, Washington, D.C. 20057, USA.

${ }^{\dagger}$ Correspondence should be directed to colin.carlson@georgetown.edu.

October 30, 2020

\begin{abstract}
Infections with parasitic worms, or helminthiases, are a class of neglected tropical diseases that affect at least one billion people worldwide. While some infections are asymptomatic, others can cause severe health conditions such as blindness, epilepsy, and cancer. The burden of these conditions disproportionately affects people in resource-poor areas, making many helminthiases chronically understudied. Mapping helminth outbreaks, prevalence, and habitats in affected regions can offer valuable insights into the spatial scope and burden of these infections. In this study, we conducted a systematic literature review of 232 helminth species that have been recorded infecting humans. Selected out of 6,829 abstracts, we analyzed 485 mapping studies documenting, inferring, or predicting the spatial scope of 45 helminth species. We use these studies to identify hotspots and coldspots of research effort, to highlight gaps in spatial data for particularly harmful or prevalent species, and to collect information about co-occurrence and syndemics among helminthiases and with other diseases. We finally suggest ways these findings could help direct future mapping studies, and ultimately inform public health interventions in understudied and underfunded regions.
\end{abstract}




\section{Introduction}

Helminths are a polyphyletic group of parasitic worms capable of infecting a variety of hosts, including wildlife, livestock, and humans. Of the roughly 350,000 species of helminth parasites of vertebrates (1), a few hundred can either complete their life cycle in humans or incidentally cause disease in paratenic human hosts. The human helminthiases exhibit a tremendous diversity of epidemiology, pathology, and ecology. Best known are the soil-transmitted helminths like hookworm, which are directly transmitted via the fecal-oral route or transdermally (often through bare feet); other species rely on arthropod or gastropod vectors, or are food- or water-borne. Most helminth species that infect humans colonize and reproduce in the gut epithelium, but some species can migrate to the liver, lungs, brain, bladder, eyes, or other tissues, causing a range of pathologies comparable in diversity to bacterial or viral infections.

Human helminthiases have a massive global burden. An estimated 1.45 billion people worldwide are infected with soil-transmitted helminths, (2) and these infections collectively account for over 3.3 million disability-adjusted life years (DALYs). (3) These may be underestimates, given more recent estimates that hookworm alone may account for more than 4 million DALYs. (4) While not a prominent cause of death, some of the most common helminth infections (including hookworm, schistosomiasis, ascariasis, and trichuriasis) put children at high risk of stunted growth and potentially impaired cognitive development (5). Moreover, helminth infections can predispose susceptibility to co-infections with other diseases (6), which can produce worse clinical outcomes, as can antagonistic immune interactions between helminthiases and treatment of other diseases (iatrogenic syndemics (7)). Other, rarer helminthiases cause acute illness and life-threatening chronic health conditions in isolation: onchocerciasis is the second leading global cause of blindness, Taenia solium infections are a leading cause of adult-onset epilepsy (ㅁ), schistosomiasis can cause bladder cancer (9), and some food-borne trematodiases can cause liver or bile duct cancer. Despite the severity of these conditions, helminthiases dominate the World Health Organization's official list of neglected tropical diseases; almost all of these conditions are more often framed as chronic health burdens and background conditions of poverty than as serious, acute epidemics.

The burden of helminth infections is highly heterogeneous over space, from case clustering at the community level up to the highly-uneven global distribution of helminthic diseases. The highest-burden infections - like schistosomiasis, hookworm, and lymphatic filariasis - are disproportionately prevalent in Sub-Saharan Africa, tropical Latin America, and South Asia. In these regions, incidence is often highest among young children, and in adults among rural poor livestock keepers, fishers, and other communities that live and work close to environmental reservoirs of transmission. (10) Furthermore, severe symptoms of helminthiases are more likely to manifest in individuals with a high worm burden, or individuals whose infections have gone untreated for years, leading to long-term disability. Infections persist and recur disproportionately where healthcare systems are too limited for routine treatment, preventative therapies, and case management; but these areas are, conversely, often the places where disease surveillance is most limited, and so the burden of these infections is most poorly characterized.

Many previous studies have therefore identified geospatial analysis, including both descriptive mapping and spatial modeling, as a key part of scientific and clinical work on helminthiases. These projects add at least five major types of value to research efforts. First, and most fundamentally, maps can be a simple, easy way to visualize data, communicate risk, and engage local communities in participatory research methods. Second, geospatial modeling can help fill knowledge gaps about the prevalence or incidence of infectious diseases. Doing so usually requires researchers 
to synthesize smaller-scale data that may not have been intended for reuse, but gain new life in a synthetic format (another incidental benefit). Using these data, researchers can develop inferential models with predictive capacity in less-sampled regions, turning isolated clinical work into a more continuous view of transmission; with enough data, this approach can be used to translate local prevalence data into regional and global estimates of incidence or burden. The process of quantitative modeling can also help identify environmental and social risk factors, which can support efforts to identify vulnerable communities that are undersurveilled, or to project changing transmission risk in the future.

Finally, and perhaps most importantly, geospatial work helps target and improve interventions: mapping can help researchers understand where interventions are most needed, where the coverage of healthcare is inaccessible or insufficient, and where interventions have had the most and least success interrupting transmission. The vast majority of interventions have focused on a half-dozen or so infections (the soil-transmitted helminthiases, schistosomiasis, lymphatic filariasis, and onchocerciasis), and therefore, so have most mapping projects. Since the turn of the century, the World Health Organization (WHO) and international development organizations have made major investments in mass drug administration (MDA) programs, which aim to control disease burden, prevent new infections, and eventually support the elimination of these infections in targeted regions. Using data from MDA programs, the distribution and burden of these diseases has been well-characterized by the Global Burden of Disease studies, the Global Atlas of Helminth Infection (www.thiswormyworld.org), and several MDA-specific national mapping programs.

Beyond the infections that have been targeted by elimination campaigns, much less is known. In some cases, this reflects formal priorities set by global health programs: helminth infections that are primarily benign, or thought to be rare, are considered lower priority for research and surveillance efforts than those that are highly prevalent or pathogenic. However, these assessments are informed by global incidence and burden estimates that - somewhat circularly - may be distorted by data deficiencies. In some cases, back-of-the-envelope global incidence estimates or distribution maps have not been updated for decades due to underreporting and limited research, even for highly prevalent and highly pathogenic infections. For example, conditions like neurocysticercosis (Taenia solium) and baylisascariasis (Baylisascaris procyonis) have high case fatality rates, but have a correspondingly limited evidence base for their distribution and prevalence, leading to low but uncertain estimates of their global burden.

While mapping is foundational to global health research, little work has examined the balance of research effort and systematic bias in these efforts as they broadly apply to helminthiases. A systematic analysis of research trends could help identify the limitations of existing data, target interventions more effectively, and broaden the scope of helminth research and control. In this paper, we set a baseline on the work mapping human helminthiases that has been published to date. To do so, we performed a systematic literature review of over 6,000 candidate mapping studies on a total of 232 helminth species known to infect humans, and we built a database of studies that perform mapping work at a wide range of scales and methodologies (see Methods). With this database, we identify global and regional hotspots and coldspots of research effort; summarize current knowledge about co-occurrence and syndemics among helminthiases and with other diseases; highlight gaps in spatial data for particularly harmful, prevalent, or otherwise significant species; and identify a list of neglected helminthiases that should be re-prioritized. Our findings could help direct further helminth mapping efforts and inform future public health interventions in understudied and underfunded regions. 


\section{Most helminth species are unmapped or undermapped}

We found a total of 485 studies that included a novel scientific component mapping a human helminthiasis across different scales, regions, pathogens, and purposes. We observed that a small number of conditions account for the vast majority of mapping studies (Figure 1). In large part, schistosomiasis, the soil-transmitted helminthiases (hookworm, Ascaris, and Trichuris), and lympathic filariasis account for the majority of studies in our database. Of the 232 species we searched for studies on, only 45 had any associated mapping studies - indicating nearly 200 conditions are mostly unmapped with scientific methods.

The simplest explanation for this uneven research effort is that a small handful of highly-prevalent infections account for the majority of the total burden of helminthiases on global health and poverty. Ascariasis is the most common helminth infection in the world, thought to infect between 737 million and 872 million people worldwide. (11) Heavy Ascaris infections can cause intestinal blockage and stunted growth in children, and ascariasis-related complications may be responsible for up to 60,000 deaths per year. (12) Other soil-transmitted helminthiases with similar pathologies like trichuriasis and hookworm infection are thought to be similarly prevalent, infecting roughly 435 million and 450 million people worldwide, respectively. (11) Meanwhile, between 179 million and 200 million people worldwide are thought to have schistosomiasis (11), which exacts a high burden especially in HIV-coendemic areas; the WHO estimates that 24,000 people died of schistosomiasis in 2016. (13) Finally, lymphatic filariasis (Wuchereria bancrofti and Brugia spp.) is thought to infect between 24 million and 36 million people. (11) Severe cases of lymphatic filariasis may cause lymphoedema, hydrocele, and adenolymphangitis, conditions that are widely stigmatized and may render patients disabled. (13)

\section{Feedbacks between mapping and interventions}

The priorities of the field to date also reflect feedbacks among mapping work, ease of treatment, and scale of interventions. A small number of infections are targeted by MDA programs, both because of cheap widely-available treatments and because they account for the highest global burden. These programs are naturally complimentary with spatial analysis: defining the boundaries of a community, testing people or animals for helminthiases, and updating endemicity maps is one of the easiest ways to visualize current burden and decide on the frequency of drug administration. This ongoing feedback of prevalence studies, GIS work, and targeted drug administration has been a key part of successful MDA efforts over the past 20 years, not just to tailor efforts but also to measure their success and justify ongoing funding. These programs are therefore the main reason that broad, synthetic data and cartography are possible for a small subset of the best-mapped helminthiases (i.e., soil-transmitted helminths, schistosomiasis, lymphatic filariasis, and onchocerciasis). Conversely, we found that most infections without readily available antihelminthic treatments were relatively understudied, or never appeared in our dataset.

Another closely-linked pattern we observed was that many under-represented helminth species were zoonotic, probably because their complex life cycles and other hosts make them more challenging targets for surveillance. For example, soil-transmitted helminths like Ascaris lumbricoides and hookworm have a relatively simple life cycle, making them easier to study; prevalence data in humans are usually likely to capture the extent of transmission (but see (14)). (Soil sampling is nearly never implemented for mapping efforts; Figure S1.) When non-human hosts are studied, they are almost always livestock (especially cattle and sheep), pets (cats and dogs), or synanthropic 
wildlife (rats and mice); true wildlife hosts account for a small fraction of studies (Figure 2). Some of the most understudied helminthiases are the ones that complete parts of their life cycle in hosts that are particularly difficult to study, like fish (e.g., Anisakis or Diphyllobothrium). Similarly, vector-borne helminthiases add another layer of ecological influence, which can make ecological models more useful, but primary data collection more challenging; we found more studies collected spatial data on schistosomiasis in snails than on more mobile vectors like mosquitoes or flies.

Across conditions, we observed a gradient between two general profiles of helminthiases: wellmapped infections, with a simple life cycle and a high burden, readily treated with MDA; and poorly-mapped infections, with a complex zoonotic life cycle, a medium or uncertain burden, and few vertical control programs. Zoonotic pathogens are usually harder to manage through vertical interventions, especially if non-human reservoirs maintain infection, and it is possible that this has lowered the collective priority on mapping these pathogens. However, we suggest that efforts could be redoubled to engage in mapping work that supports One Health interventions geared toward vector control, community sanitation, food safety, livestock vaccination, routine deworming of household pets, and similar practices - especially for less-treatable conditions. For example, Dirofilaria infections can only be treated with surgery (15), but as with other mosquito-borne diseases, mapping efforts can help predict spatial patterns of risk, identify and eliminate spatial risk factors (like standing water), and promote and target the use of insecticides. Similarly, Taenia solium neurocysticercosis is largely incurable, but One Health interventions that pair MDA for taeniosis with pig vaccination can eliminate the pathogen over 4-5 years (16); given that porcine infection control programs are globally underfunded, better data on the distribution of hyperendemic communities can help allocate investments for the greatest returns. Although they receive less global support and funding than existing MDA programs, these One Health programs achieve a high return-on-investment by preventing infections.

\section{Mapping starts at small scales, but the field is growing}

The number of helminth mapping studies has steadily increased since the year 2000, and the field is still growing rapidly. Across all time periods, we found that most studies use maps as a data visualization tool (case occurrence data or prevalence maps; Figure 3); however, the last decade has seen a particular shift towards advanced statistical modeling and machine learning approaches. In particular, tools from ecological niche modeling began to be used around 2007 to 2010, when the most popular algorithms (MaxEnt and GARP) began to cross over into medical geography. This particular approach to predictive modeling is continuing to become more popular as more disease ecologists become involved in neglected tropical disease research. In the last decade, we also observed a methodological shift away from studies using licensed software like ArcGIS, and increasingly taking advantage of open-source software like QGIS and GRASS, or console programs like R and Python. These accessible softwares can be easily used by researchers and stakeholders without the financial barriers of proprietary softare that are prohibitive even for many in the Global North.

The vast majority of the studies in our database were conducted in a single community or a handful of communities in a single country (Figure 3). For most disease mapping efforts, these types of community prevalence studies form the basis for geographically-broader risk maps and other forms of predictive spatial modeling. With exceptions for the best-studied pathogens (soil-transmitted helminths, schistosomiasis, and lymphatic filariasis), we found relatively few large-scale (multinational to global) prevalence or risk maps, likely because the raw prevalence 
data needed to comprehensively and accurately create them is deficient for many infections. Given the shortage of global helminth maps, it is likely that global prevalence estimates for many helminth species are based on incomplete prevalence data, if these estimates exist at all. Reliable global prevalence estimates and comprehensive global prevalence and risk maps are needed to assess the true burden of helminthiases and inform the allocation of resources and funding.

Therefore, it is critical that future small-scale helminth surveillance efforts be conducted with large-scale mapping efforts in mind, surveying regions that have been historically neglected. Our analysis revealed that entire regions appear to be understudied (Figure 4p: several countries in Southeast Asia, Latin America, and the Caribbean are known to have high parasitc burdens, but have relatively few mapping studies compared to China, Brazil, and tropical Sub-Saharan Africa. Countries like China and Brazil might have more mapping studies than other countries in their respective regions as a function of their size, population, and sometimes their unique community of practice in medical parasitology (e.g., Fiocruz in Brazil). However, other populous nations lack mapping studies that accurately reflect their helminth burden: for example, soil-transmitted helminth prevalence exceeds $50 \%$ in some parts of India (17), but data is clearly lacking proportional to the country's population and size. It seems highly plausible that gaps between expert opinion and available data about the burden of some infections (e.g., the apparently low global burden of T. solium neurocysticercosis and epilepsy) is largely driven by these global gaps in surveillance, reflecting the priority systems and Balkanization of aid programs and health financing.

\section{Putting the burden of helminthiases in context}

Because many different helminthiases share socioecological drivers, their burden is often highest in the same communities. Co-infection with multiple helminthiases can exacerbate clinical outcomes or even produce severe disease, a syndemic interaction that makes local burden higher than expected if each disease existed in isolation (18). Knowing where helminthiases are co-endemic with each other - or even better, where co-infection intensity is highest - can help guide synergistic public health interventions that target several infections at once, like MDA. Often, these data are already collected by surveillance systems: for example, monitoring of schistosomiasis, Ascaris, and Trichuris is often synergistic, as all three can be easily screened using the sample samples with the Kato-Katz method (while hookworm is slightly more challenging). Similarly, three studies in our database surveyed communities for Schistosoma mansoni and hookworm co-infections. In all three studies, survey participants who were co-infected or at high risk of co-infection were able to treated with both praziquantel and albendazole for S. mansoni and soil-transmitted helminth infections (STH), respectively. (19; 20; 21)

Spatial approaches also often address the inefficiencies, limitations, and pitfalls of MDA. For example, co-infection can render some standard anti-helminthic treatment protocols ineffective or even lethal. Most notably, treatment of patients with a Loa loa infection with ivermectin (the most widely-used antifilarial drug) can cause severe adverse events. Hundreds of cases of severe neurological complications and several deaths have been reported, and caution in MDA has been widely recommended. (22) Seven studies in our dataset mapped where Loa loa and Onchocerca volvulus are co-endemic in West and Central Africa to identify communities where ivermectin-based onchocerciasis control programs should be deployed with caution or avoided altogether. Continued surveillance to identify co-infection hotspots helps MDA programs monitor where ivermectin can be administered safely, and where other interventions like vector control 
Chronic helminthiases frequently exacerbate other health conditions or increase susceptibility to additional infections, further broadening the potential impact (and necessary scope) of mapping efforts. In our review, we found 14 studies that mapped where helminthiases and other diseases are co-endemic or where individuals are jointly infected with helminths and other pathogens. For example, three studies mapped malaria co-infections with hookworm (21, 23, 24), two additional studies mapped co-infections with schistosomiasis (25, 26), and another examined co-infections with lymphatic filariasis (27). These helminthiases are commonly comorbid with malaria in sub-Saharan Africa and the Indian subcontinent. Little is known about the interactions between these parasitic species, and there is conflicting evidence as to whether the anti-inflammatory immune response induced by helminth infections makes individuals more susceptible to malaria or confers protection against severe malaria. Some studies suggest that this protective effect may be exclusive to certain helminth species: while individuals with $S$. mansoni or hookworm infections may be more susceptible to malaria, S. haematobium infections may confer protection against severe malaria in individuals asymptomatically infected with Plasmodium falciparum. (28; 29) While this may reduce the apparent severity of disease, it could also reduce the efficacy of surveillance for febrile illness, leading to higher undetected malaria transmission.

As malaria is still a leading cause of mortality in Sub-Saharan Africa, identifying hotspots of helminthiases that suppress the anti-malarial immune response could guide interventions that jointly reduce the burden of helminth infections and malaria deaths (30; 31). MDA programs may be able to expand treatment in populations with high co-infection risk for malaria and schistosomiasis by pairing praziquantel, an anti-helminthic drug that targets adult worms, with artemisinin, an anti-malarial drug that can target both Plasmodium falciprarum and immature schistosomes (32); the timing of these interventions can be structured around malaria seasonality, pointing to the value of spatiotemporal analyses (30). Moreover, recent studies suggest that the ivermectin might reduce the lifespan of Anopheles mosquitoes that bite those who have been treated (33, 34), potentially expanding the impact of "rapid impact" packages as a supplemental malaria control measure. (35) In addition to MDA, vector control efforts can be synergistically targeted to reduce both malaria and helminthiasis transmission, especially lymphatic filariasis. One study in our literature review mapped where individuals are co-infected with Wuchereria bancrofti and Plasmodium falciparum in Uganda, noting the same species of Anopheles mosquito carries both pathogens in the region. (36) Little is known about how $W$. bancrofti and $P$. falciparum interact in co-infected individuals, but efforts to control Anopheles mosquitoes in regions where this vector is a carrier for both parasites could nonetheless significantly reduce the burden of both conditions, especially paired with ivermectin. Mapping Anopheles vectors in this region can help target joint control efforts and trial new approaches to MDA.

More broadly, understanding where these kinds of co-infections occur could maximize the impact of helminth control programs, not just on helminthiasis burden but on the broader burden of infectious diseases of poverty. Despite coarse work on co-endemicity, no studies in our literature review mapped individual rates of co-infection for helminthiases and a handful of conditions, like HIV and tuberculosis, that are known to cause severe disease together. As researchers discover more about how helminthiases interact with each other, with other pathogens, and with the drugs used to treat them, the need for spatial data showing where these interactions are most significantly causing harm becomes more apparent. Furthermore, as researchers continue to discover novel interventions that safely treat multiple pathogens at once, spatial data showing where these interventions can be deployed most effectively will be needed. 


\section{Priorities for future work}

We found that there is little information about the prevalence, incidence, and spatial distribution of most helminth species. These infections range from sporadic, paratenic infections to global burdens, and many represent a small, unquantified, or highly local burden; however, a handful of noteworthy infections remain understudied and unmapped. These gaps are unlikely to change without a corresponding change in data collection: of over 200 helminthiases we searched for, most have no coordinated surveillance system at all, and as a result, many are data deficient to a degree that precludes mapping efforts. However, the existence of globally-coordinated programs to track and manage soil-transmitted helminths and schistosomiasis - and to eliminate lymphatic filariasis, onchocerciasis, and Guinea worm disease - suggests that similar scientific and clinical coordination is broadly possible for other infections.

Based on the gaps we have identified, we propose a candidate list of helminthiases which may particularly benefit from additional mapping efforts (Box 1). Our list of possible priorities reflects an informal assessment of which infections have some combination of a high global burden, high case fatality rate, and high spatial heterogeneity in incidence (including a strong ecological component). We also prioritized infections that are not readily treated by the same MDA programs that are already in place throughout much of sub-Saharan Africa and southeast Asia, on the grounds that mapping efforts can help estimate incidence, and target treatment efforts or One Health prevention efforts focused on zoonotic and vector-borne transmission.

Box 1. Proposed list of high-priority neglected helminthiases for research and policyoriented mapping, in alphabetical order. (*Guinea worm disease is not comparatively neglected but deserves special mention due to important questions about its enzootic cycle.)

1) Angiostrongyliasis (Angiostrongylus cantonensis)

2) Hepatic and intestinal capillariasis (Capillaria hepatica, Capillaria philippinensis)

3) Carcinogenic food-borne trematodiases (Clonorchis sinensis, Opisthorchis viverrini)

4) Guinea worm disease* (Dracunculus medinensis)

5) Echinococcosis (Echinococcus granulosus, E. multilocularis)

6) Gastrodiscoidiasis (Gastrodiscoides hominis)

7) Dwarf tapeworm (Hymenolepis nana)

8) Mansonellosis (Mansonella perstans, M. ozzardi, M. streptocerca)

9) Strongyloidiasis (Strongyloides stercoralis)

10) Taeniosis and cysticercosis (Taenia solium)

We divided our final priority list into three categories, which we review in turn: (1) Infections of notable significance but an uncertain global burden, which could be readily estimated given a coordinated data synthesis and modeling effort that leverages existing work; (2) Infections with 
a global distribution, which could be readily mapped given a coordinated data synthesis effort; and (3) Infections with a high local burden that still require first-principles, community-based prevalence mapping studies to set an epidemiological baseline. The list is subjective, and far from comprehensive, but offers a starting point for expanding surveillance priorities.

\section{Opportunities for global burden estimation}

Taeniosis and cysticercosis (Taenia solium): Several species of Taenia can infect humans, but the most notable is Taenia solium, a zoonotic parasite with a pig intermediate host, most common in communities with poor sanitation where people eat raw or undercooked pork. Intestinal infection with the adult tapeworm (taeniasis) is often asymptomatic or mild. However, fecal-oral transmission between humans can allow eggs to develop into larvae and form cysts in human tissues (cysticercosis). When these cysts form in the brain or on the spinal cord (neurocysticercosis), the resulting condition is particularly severe. (13) While T. solium frequently co-occurs with two closely related tapeworms (the beef tapeworm T. saginata, and the Asian tapeworm T. asiatica), the former is not known to cause neurocysticercosis, and the latter's status remains controversial.

T. solium has been reported worldwide, but has the highest incidence in Latin America, Eastern Europe, sub-Saharan Africa, China, and southeast Asia. No consolidated global map of Taenia solium exists, and global burden estimates for taeniasis and cysticercosis vary substantially across sources. The WHO estimates that 5.5 million people have T. solium infections (13), and the 2016 Global Burden of Disease Study estimates that between 2.2 million and 3.1 million people have cysticercosis. (11) Furthermore, the WHO estimates that T. solium infections are responsible for 28,000 deaths annually. (13) However, these are likely underestimates, as regional burden estimates are similarly high (e.g. 1.2 million estimated cases of epilepsy due to neurocysticercosis in India alone) (37). Moreover, neurocysticercosis is a leading cause of preventable epilepsy worldwide, with one review estimating that neurocysticercosis may be responsible for $31.5 \%$ of epilepsy cases in regions where T. solium is endemic. (38) A global reassessment of the burden of neurocysticercosis in particular might help motivate greater support for control (especially pig vaccination and improved sanitation) and treatment (MDA), as well as coordinated regional partnerships to eliminate the infection.

Strongyloidiasis (Strongyloides stercoralis): Strongyloides stercoralis is a soil-transmitted nematode that is common worldwide in rural communities lacking proper sanitation. Strongyloidiasis is often asymptomatic, but can be life-threatening in immunocompromised individuals, such as transplant recipients, or people with HIV or hematologic diseases like leukemia. (39) The WHO estimates that 30-100 million people are infected worldwide, though they acknowledge that this is likely an underestimate (40); prevalence maps have been developed at national levels, but many gaps in surveillance exist. (41) Our literature review identified just three efforts to map this parasite - all of which were small-scale national or community studies - highlighting a need for more community-based surveillance efforts, and an effort to consolidate existing data into a better global dataset for finer-scale mapping. Additionally, given that HIV might exacerbate strongyloidiasis, identifying regions where these conditions are syndemic might help target interventions more effectively, like the eastern Mediterranean region (42; 43).

Dwarf tapeworm (Hymenolepis nana): Dwarf tapeworm infections are some of the most common human cestode infections, and are particularly prevalent in children. While most dwarf tapeworm infections are asymptomatic in adults, prolonged infections (caused by self reinfection 
within the intestine) can cause a variety of symptoms, such as nausea, dizziness, weakness, and abdominal pain. (44) These symptoms are more common in children and may be more severe when coincident with malnutrition. (45) H. nana infections are more prevalent in warmer climate zones, but are also found in temperate regions of Europe, Asia, and the Americas. Very little is known about $H$. nana's global prevalence and distribution, and regional prevalence estimates vary significantly. For example, between 0.2 and $28.4 \%$ of the population of Asia is thought to have $H$. nana infections, and between 0.9 and $23 \%$ of the population of the Americas is thought to be infected. (46) Our literature review found only two studies on H. nana, both community-based studies in Angola and Ghana, respectively. (23, 47) Future surveillance efforts should aim to establish reliable prevalence and incidence estimates in regions where $H$. nana is highly endemic and where $H$. nana infection and malnutrition are likely to be comorbid. These efforts could support the development of a global prevalence map, and help direct future MDA efforts.

\section{Opportunities for global distribution or risk mapping}

Angiostrongyliasis (Angiostrongylus cantonensis): Humans are a paratenic host for $A n$ giostrongylus cantonensis, usually becoming infected after eating snails or slugs infected with $A$. cantonensis larvae or vegetables contaminated with mucus from infected snails or slugs. Some cases of angiostrongyliasis (also called rat lungworm) are asymptomatic, but A. cantonensis larvae can burrow into neural tissue, causing eosinophilic meningitis. (48) People with this condition may experience symptoms such as headaches, neck pain, nausea, and vomiting for several weeks until the parasite dies and the infection resolves itself. (49) However, high worm burdens may cause more severe presentations, including significant neurological damage and death. (48)

Angiostrongyliasis is most common in southeast Asia and the Pacific islands, and it is the most common cause of eosinophilic meningitis in south Asia. However, climate change and increased migration to and from endemic areas are facilitating the expansion of its geographic range beyond the tropics, hence its status as an emerging infectious disease. (48; 49) Angiostrongyliasis is notably very difficult to diagnose, and current treatments are only able to lessen the severity of symptoms. Furthermore, at least 2,800 cases have been reported to date (49), but global estimates of incidence remain limited. Our literature review identified two A. cantonensis global prevalence maps; smaller-scale mapping efforts could help identify where preventive public health interventions would be most effective.

Echinococcosis (Echinococcus granulosus and E. multilocularis): Echinococcus granulosus and Echinococcus multilocularis are zoonotic tapeworms that cause cystic and alveolar echinococcosis, respectively. Humans can contract echinococcosis by ingesting food contaminated with the feces of infected dogs or foxes. These infections typically remain asymptomatic for years until cysts grow large enough to disrupt organ function. For example, E. granulosus cysts in the lungs can cause a chronic cough and chest pains, while cysts in the liver caused by $E$. multilocularis often manifest as destructive tumors that cause abdominal pain, biliary obstruction, and hepatomegaly. (13) These cysts have the potential to rupture, causing a high fever and even inducing anaphylactic shock; as a result, echinococcosis has a relatively high case fatality rate. (50)

The WHO estimates that around 1 million people have echinococcosis. These infections end in roughly 19,000 deaths per year, with alveolar echninococcosis having a significantly higher case fatality rate due to its potential to cause liver failure when left untreated. (13) Anti-helminthic drugs have limited effectiveness against Echinococcus infections when used in isolation, and 
infections are more commonly resolved by surgically removing cysts and initiating patients on long-term chemotherapy. (50) Our literature review did not identify a global prevalence or risk map for echinococcosis, though a global synthetic prevalence map has recently been published (51); finer-scale prevalence mapping remains an important task, and can be accomplished using popular species distribution modeling methods. While these helminths are found worldwide, most of the mapping efforts we found were community studies in Western Europe and China. Therefore, it is critical that future surveillance efforts aim to identify more echinococcosis hotspots where preventive public health interventions could be effective.

Mansonellosis (Mansonella perstans, M. ozzardi, M. streptocerca): Mansonellosis is a vector-borne infection spread by Culicoides midges. $M$. ozzardi and $M$. streptocerca develop and reproduce in the skin, while $M$. perstans migrates to body cavities after transmission and develops there. Many mansonellosis infections are asymptomatic, and symptoms that do arise are typically mild. However, eosinophilia is a common symptom of $M$. perstans infections, and other symptoms such as fever, rashes, headache, and joint pain have been observed. (52) $M$. perstans alone is thought to infect over 100 million people, mostly in Sub-Saharan Africa and South and Central America. However, anti-helminthic drugs have limited effectiveness at treating mansonellosis, particularly $M$. perstans infections. (52) Furthermore, despite the high global burden of mansonellosis, there are currently no large-scale control programs targeting Mansonella species. Our literature review identified nine studies mapping Mansonella species, including one global endemicity mapping study. Future research could aim to generate a global mansonellosis risk map to guide vector control efforts, particularly given that Culicoides midges also transmit several emerging infections (including bluetongue virus, Oropouche virus, and African horse sickness).

Guinea worm disease (Dracunculus medinensis): Unlike other infections on our shortlist, Guinea worm disease cannot be considered neglected; decades of control efforts have brought Guinea worm disease close to being the first globally-eradicated parasitic disease. In 2019, only 53 cases were reported from four countries (South Sudan, Angola, Cameroon, and Chad). (13) Despite the small number of remaining foci, D. medinensis was once found throughout Africa, Latin America and the Caribbean, the Middle East, and southern Asia. Little geospatial data has been consolidated on this century-long range contraction; the best available maps of its original range are hand-drawn estimates from the 1950s. (53) Remapping the historical distribution of $D$. medinensis with modern technology and modeling methods could offer some insights into the previous successes of eradication campaigns, and offer fresh motivation to eliminate Guinea worm disease on the last remaining continent. This may be particularly useful given emerging evidence of the scope and extent of the enzootic cycle, with confirmed animal cases in countries that might otherwise be approaching elimination. (54, 55) Geospatial data has already been a critical tool for understanding the scope of enzootic transmission; further ecological modeling could offer some insights into the possible extent of the zoonotic niche, especially given the once-broad historical range of the parasite.

\section{Opportunities for regional and community-based mapping}

Carcinogenic food-borne trematodiases (Opisthorchis viverrini and Clonorchis sinensis): $O$. viverrini and $C$. sinensis are liver flukes that can infect humans in their metacercarial stage. Humans contract these infections by eating fish infected with metacercari. (56) Most $O$. viverrini and $C$. sinensis infections are asymptomatic, but some cause prolonged bile duct inflammation that may progress to liver cancer or bile duct cancer (cholangiocarcinoma). (56) $O$. viverrini is found primarily in northeast Thailand, the Lao PDR, Cambodia, and central and

\section{.}

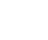

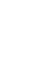

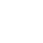

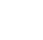

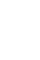

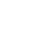

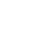

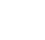
.

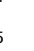


medRxiv preprint doi: https://doi.org/10.1101/2020.10.30.20223529; this version posted November 4, 2020. The copyright holder for this preprint (which was not certified by peer review) is the author/funder, who has granted medRxiv a license to display the preprint in It is made available under a CC-BY-NC-ND 4.0 International license .

southern Vietnam. It is most prevalent in Thailand, where it costs the country an estimated 120 million USD annually and is found in up to $70 \%$ of people in some communities. (56) Northeast Thailand also has the highest incidence of cholangiocarcinoma in the world: 85 people for every 100,000 develop the condition per year. (57) C. sinensis infects an estimated 35 million people worldwide, predominantly in China, Korea, Vietnam, Taiwan, Thailand, and Russia. Regions where $C$. sinensis is hyperendemic have high incidences of cholangiocarcinoma, and both $C$. sinensis infections and cholangiocarcinoma cases are on the rise in these regions. (58) Given that our literature review did not identify any community-based mapping efforts for $C$. sinensis and identified very few community-based mapping efforts for $O$. viverrini, it is critical that researchers undertake more of these small-scale studies.

Capillariasis (Capillaria hepatica and Capillaria philippinensis): C. hepatica and C. philippinensis cause hepatic and intestinal capillariasis, respectively. Humans contract C. hepatica by ingesting embryonated eggs from fecally contaminated food, water, or soil. Larvae migrate to the liver parenchyma to develop, mate, and reproduce (59); eggs remain in the liver parenchyma until the infection is treated or the patient dies. Little is known about the pathology of C. hepatica infections, but it has been known to cause severe and potentially fatal liver disease in human hosts when left untreated. Furthermore, C. hepatica infections are notoriously difficult to diagnose, as routine laboratory tests fail to identify the parasite, which is usually only diagnosed via biopsy or necropsy. (59) Humans are usually exposed to C. philippinensis by eating fish infected with the larvae, which develop and reproduce in the intestinal mucosa (60), causing abdominal pain, weight loss, and diarrhea. If left untreated, infected individuals may lose proteins and electrolytes to the point where they become severely emaciated and die. (60) Though both are apparently rare, C. philippinensis can be found throughout Asia and parts of North Africa and the Middle East, and $C$. hepatica is found in wildlife worldwide (59, 60). However, our literature search did not identify any efforts to map either species.

Gastrodiscoidiasis (Gastrodiscoides hominis): G. hominis is a poorly characterized parasitic fluke, primarily of pigs, but also humans. Humans become infected when they ingest $G$. hominis metacercaria from infected animal products or aquatic plants. The parasite colonizes the caecum, where it grows and reproduces. (61) Little is known about the mechanism of $G$. hominis infection in humans, but it can cause symptoms such as diarrhea and malnutrition. $(62$; 63) Death has been reported in some cases of heavy infection. (62) G. hominis is thought to be highly prevalent in India, but cases have been observed throughout Asia and parts of Africa. (62) Our literature search did not identify any efforts to map G. hominis infection, meaning that our understanding of who is at risk and where is severely limited. Small-scale community-based mapping efforts in areas where G. hominis infections have been reported could help direct preventive public health interventions and MDA efforts.

\section{Broader directions}

The World Health Organization recently published a 10-year "Roadmap" for neglected tropical diseases (NTDs) highlighting the successes of control programs over the past decade and establishing a new set of control priorities. (13) The report notes that ongoing efforts to control helminthiases that contribute most significantly to the global burden of poverty have been largely successful. For example, there has been a $36 \%$ reduction ( 554 million people) in the population requiring MDA for lymphatic filariasis since the Global Programme to Eliminate Lymphatic Filariasis 
was launched in 2000, and the condition has been eliminated as a public health concern in 16 countries; transmission of onchocerciasis has been eliminated in four countries in the Americas. Furthermore, $71 \%$ of school-aged children requiring preventive chemotherapy for schistosomiasis are currently receiving coverage, and $70 \%$ of pre-school and school-aged children who require treatment for soil-transmitted helminthiases are being treated regularly. (13)

The WHO roadmap presents detailed strategies to eradicate Guinea worm, eliminate transmission of onchocerciasis, and eliminate lymphatic filariasis, schistosomiasis, and soil-transmitted helminthiases as public health problems within the next decade. The plan also outlines strategies to control helminthiases that have previously lacked large-scale control programs, like echinococcosis, food-borne trematodiases, and Taenia solium cysticercosis. The WHO acknowledges that mapping these historically neglected helminthiases is a critical first step to control efforts, echoing several of the priorities for future work we have presented in this paper. (13) Given this call to action, we are optimistic that the surveillance and mapping efforts targeting these understudied helminthiases may soon be proportional to their true global burden. However, several helminthiases in our priorities for future work have yet to be widely recognized as priorities for large-scale control efforts, showing that there is substantial work left to be done.

Finally, we observe that climate change will complicate surveillance and control efforts, driving future shifts in the geographic distributions of helminths, their vectors, and their wildlife reservoirs. Ecological changes will also be met with social impacts of climate change, including weakened health systems, greater poverty, gender disparities and conflict, and an ongoing erosion of global multilateral governance. All of these are likely to interact in ways that are at times unforseeable, but which will ultimately increase the burden of neglected tropical diseases, often in new regions. Ecological niche modeling and other spatial tools can help scientists anticipate some of those potential changes: for example, the range of hookworm is projected to expand toward the southernmost regions of Africa as temperatures rise (64). The expansion of breeding ranges for lymphatic filariasis mosquito vectors and onchocerciasis blackfly vectors may also pose a significant challenge to elimination efforts in the next decade. Researchers overseeing NTD work should expect to face an uphill battle against soil-transmitted helminthiases and vector-borne helminthiases as the climate changes, scaling up MDA coverage and vector control efforts accordingly. In other cases, climate change is projected to produce local decreases: angiostrongyliasis may lose range as China warms (65), and the snail intermediate hosts of schistosomiasis may begin to shrink as their habitats grow hotter and more arid (64). Given findings like these, spatial modeling can help triage the relative added risk posed by climate change on a pathogen-by-pathogen basis. Noting how neglected most helminthiases already are, this kind of triage will be a prerequisite for researchers to demonstrate the ongoing impacts of climate change on health in the Global South, and more broadly to prepare global health for an uncertain future. 


\section{Methods}

\section{Identifying candidate species}

To compile a list of human-associated helminth species, we used a recently-published dataset of host-parasite associations curated by the Natural History Museum in London (NHM). ([66) Using this dataset, we compiled a list of human helminthiases by searching for associations with Homo sapiens, and recording the number of references listed for each species. There were 407 helminth species on this initial list. To verify whether each helminth species is still considered taxonomically valid and is capable of infecting humans, we manually searched for records of human infection for each species. In Google Scholar, we used the search queries "[species name]" and "human*" to search for records of human infection. In Google, we used the search queries "[species name]" and "syn*" to determine if species with no records or only old records of human infection have since been renamed. We removed a species from the study if we could find no evidence that the species infects humans, the species name was found to be synonymous with a more recent species name on the list, there was conflicting evidence as to whether the species can infect humans, or the species was found to infect humans only in a hybrid with another helminth species. This left a total of 232 taxonomically valid human helminthiases.

\section{Systematic review}

We used Wuchereria bancrofti as a test species to determine what search terms would be effective to pull helminth mapping studies. On Google Scholar, we used the search queries "Wuchereria bancrofti" and "mapping." Based on a handful of studies, we selected keywords that signaled spatial analysis and appeared in several studies. Our final search query was "[species name]" and ("SaTScan" or "MaxEnt" or "spatial cluster*" or "spatial analysis" or "geospatial" or "ecological niche model*" or "mapping" or "nearest neighbor" or "spatial GLM*"). We searched this set of terms alongside each of the 232 helminth species, one by one, on both PMC and PubMed. (Notably, this search may have missed grey literature led by health ministries and implementing partners, as well as studies that were otherwise missing the relevant keywords, so only provides a snapshot of published work on the field.)

For each search combination, we recorded the total number of search results each individual search generated from each database, and the number that were included after reading. We included studies if they showed novel data or a new modeling product representing the known or predicted spatial distribution of a helminth species, the condition(s) it causes, and/or the medication used to treat it. It is worth noting that since our search terms are in American English, our literature search may have missed mapping studies that were written and published in other languages.

Our literature review was conducted between November 2018 and May 2019 and excludes any helminth mapping studies published after May 2019. In total, it contains 485 human-helminth mapping efforts for 45 helminth species (see Supplementary Table 1). We followed PRISMA guidelines, and report the full process in Figure 5 . For each study in the final dataset and analysis, we recorded: the Latin names of the helminths being mapped (if specified); the citation for and link to each study; the year each study was conducted; the type of mapping used; the spatial scope of each study; the specific methodologies used in each mapping effort; the sample size and type in each study; whether each study addressed uncertainty and population at risk; whether each study examined coendemicity and/or coinfection among helminth species or between helminth species and other diseases; and whether each study had its data publicly archived. 


\section{Data ontology for study methods}

In our systematic review, we classified the mapping methodology of studies into a handful of categories, each of which are non-exclusive. More detailed categories were noted on a first pass, and then consolidated down to six types of methods:

- Grey data describes the presentation of spatial point data of either cases or positivenegative testing results. For attempts to develop post hoc databases and risk maps, these are data that could be heads-up digitized and reused (if they have not been jittered for data security and anonymization).

- Prevalence mapping refers to presenting raw or smoothed data on prevalence on a map; like grey data, this is a presentation of raw data, but with more aggregate measures of intensity. Prevalence also includes non-prevalence quantitative measures of transmission intensity, like fecal egg counts or parasite rate.

- Prevalence modeling refers to using models to unpack patterns of prevalence, involving either an explicit spatial component or spatial covariates (like climate data), or by doing spatial autocorrelation analyses like Moran's I or autocorrelograms. A binomial logistic regression that is stratified by age and sex alone would not qualify for this; such a model incorporating distance to rivers, gridded rainfall, or a conditionally autoregressive term would qualify.

- Cluster analysis refers to spatial clustering methods like SaTSCAN or directional distribution models (standard deviation ellipse), a subset of prevalence modeling focused on identifying specific, discrete points and areas of clusters or hotspots.

- Risk mapping refers to the projection of modeled risk surfaces over a continuous area or at regional levels; this involves at least some amount of inference of risk, from a model of prevalence or occurrence, and presentation of modeled results.

- Ecological niche modeling is a subtype of risk mapping, for when authors explicitly refer to habitat suitability models, ecological niche models, or species distribution models as the methodology being used. Risk maps with ecological covariates are, by definition, habitat suitability models but not all studies refer to them ecologically.

- Endemicity mapping refers to the delineation of known or suspected zones of endemicity or transmission, based on historical or published data reused or consolidated to identify likely zones. For example, this can be the identification of possibly at-risk communities, or the mapping of survey results at the national level for a whole continent. 
medRxiv preprint doi: https://doi.org/10.1101/2020.10.30.20223529; this version posted November 4, 2020. The copyright holder for this preprint (which was not certified by peer review) is the author/funder, who has granted medRxiv a license to display the preprint in

It is made available under a CC-BY-NC-ND 4.0 International license .

\section{Figures}

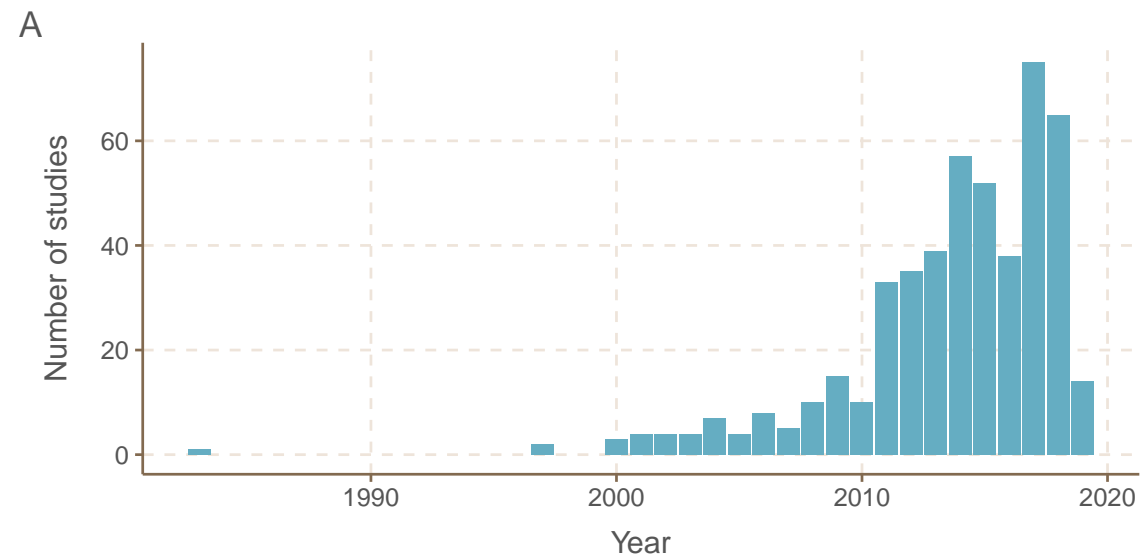

$\mathrm{B}$

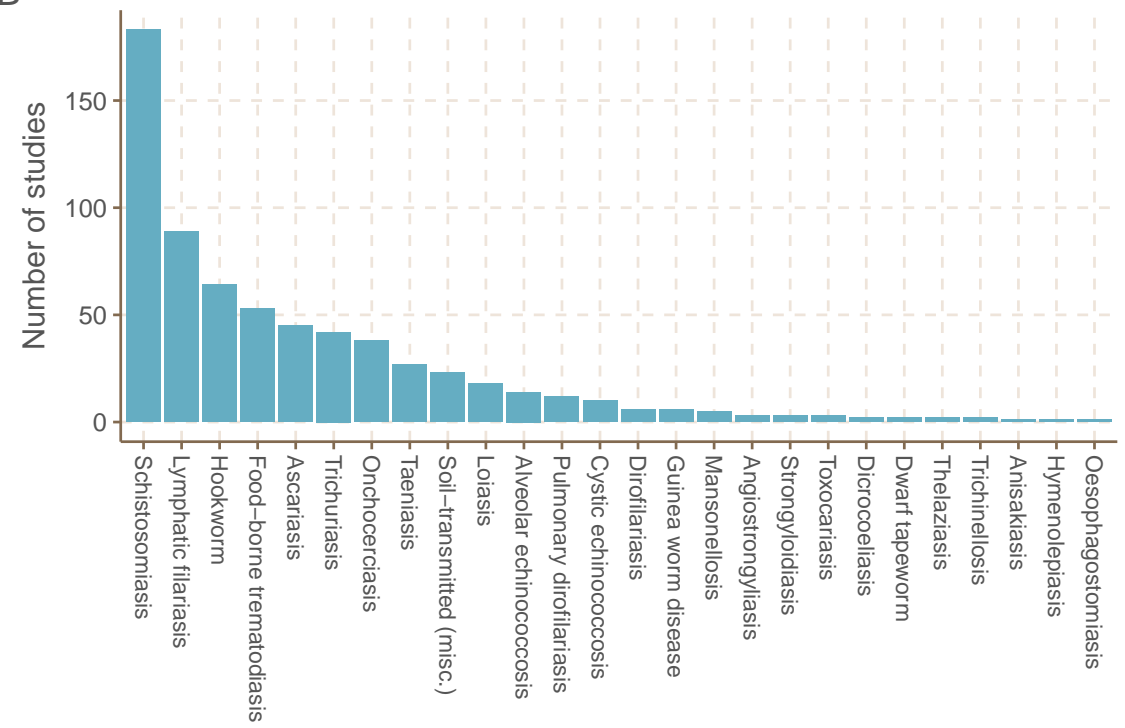

Figure 1: (A) Efforts to map the human helminthiases have increased over time. (B) Spatial data for a few helminthiases makes up the majority of all human helminth spatial data. The 45 helminth species with spatial data were grouped together by the conditions they cause (e.g. Wuchereria bancrofti and Brugia malayi grouped as lymphatic filariasis). 
medRxiv preprint doi: https://doi.org/10.1101/2020.10.30.20223529; this version posted November 4, 2020. The copyright holder for this preprint (which was not certified by peer review) is the author/funder, who has granted medRxiv a license to display the preprint in

It is made available under a CC-BY-NC-ND 4.0 International license .
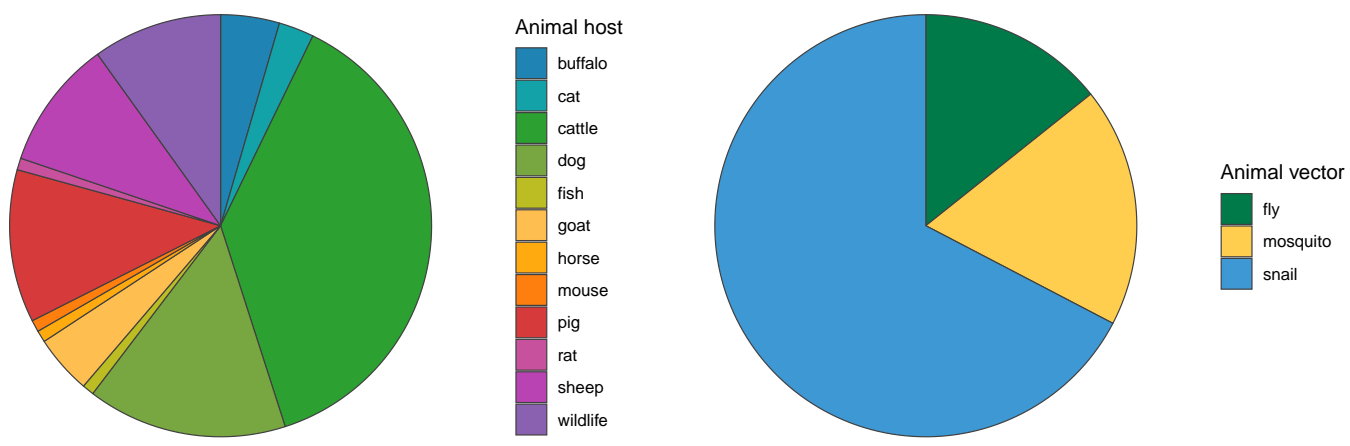

Figure 2: Among studies that map other helminth hosts or helminth vectors, studies mapping less mobile hosts and vectors predominate. Baboons, antelope, and wild boar were classified as wildlife hosts; some studies mapped multiple non-human hosts 
medRxiv preprint doi: https://doi.org/10.1101/2020.10.30.20223529; this version posted November 4, 2020. The copyright holder for this preprint (which was not certified by peer review) is the author/funder, who has granted medRxiv a license to display the preprint in

It is made available under a CC-BY-NC-ND 4.0 International license .
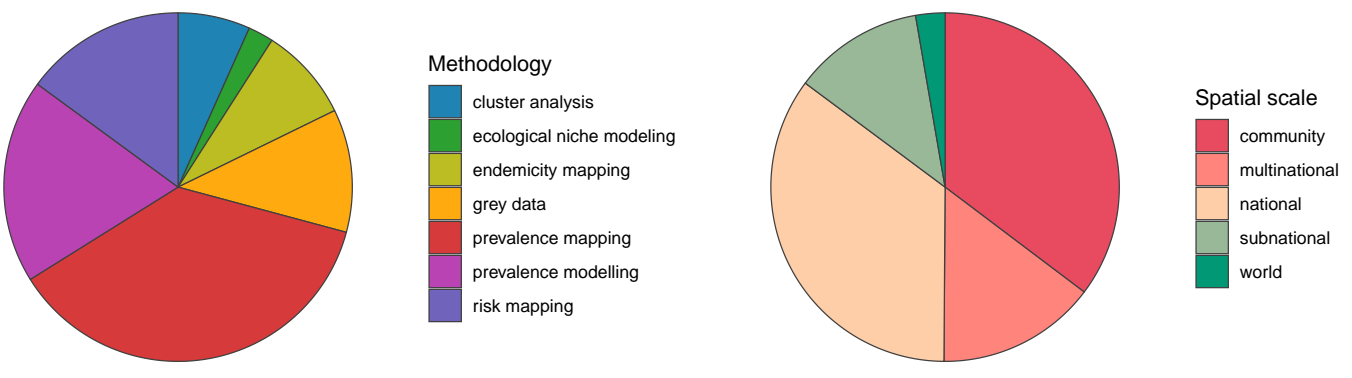

Figure 3: Existing human helminth spatial data predominantly comes from small-scale prevalence mapping studies. Studies containing spatial data on human helminthiases were characterized by spatial scale and methodology, with several studies employing more than one methodology. 
medRxiv preprint doi: https://doi.org/10.1101/2020.10.30.20223529; this version posted November 4, 2020. The copyright holder for this preprint (which was not certified by peer review) is the author/funder, who has granted medRxiv a license to display the preprint in It is made available under a CC-BY-NC-ND 4.0 International license.

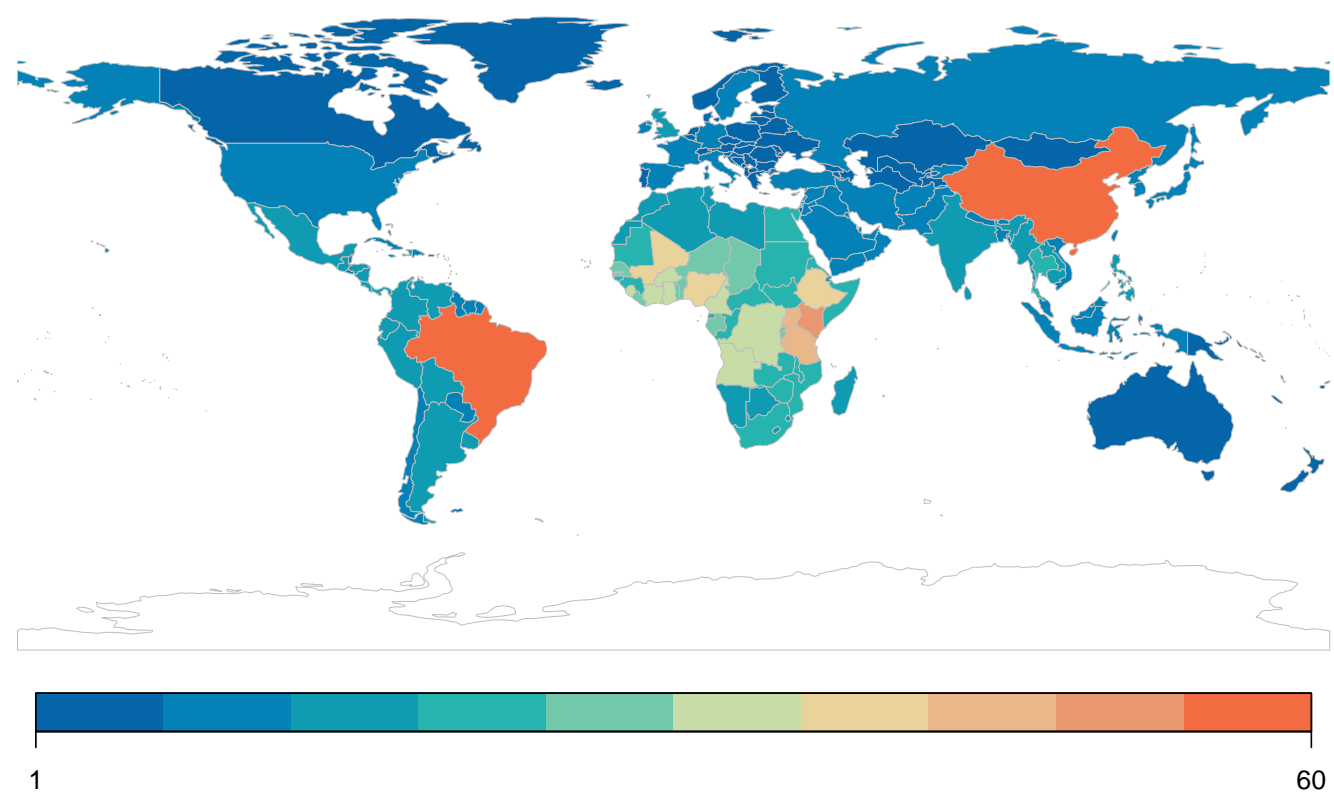

Figure 4: Most published spatial research on human helminthiases describes incidence and burden in Sub-Saharan Africa, China, and Brazil. 
medRxiv preprint doi: https://doi.org/10.1101/2020.10.30.20223529; this version posted November 4, 2020. The copyright holder for this preprint (which was not certified by peer review) is the author/funder, who has granted medRxiv a license to display the preprint in

It is made available under a CC-BY-NC-ND 4.0 International license .

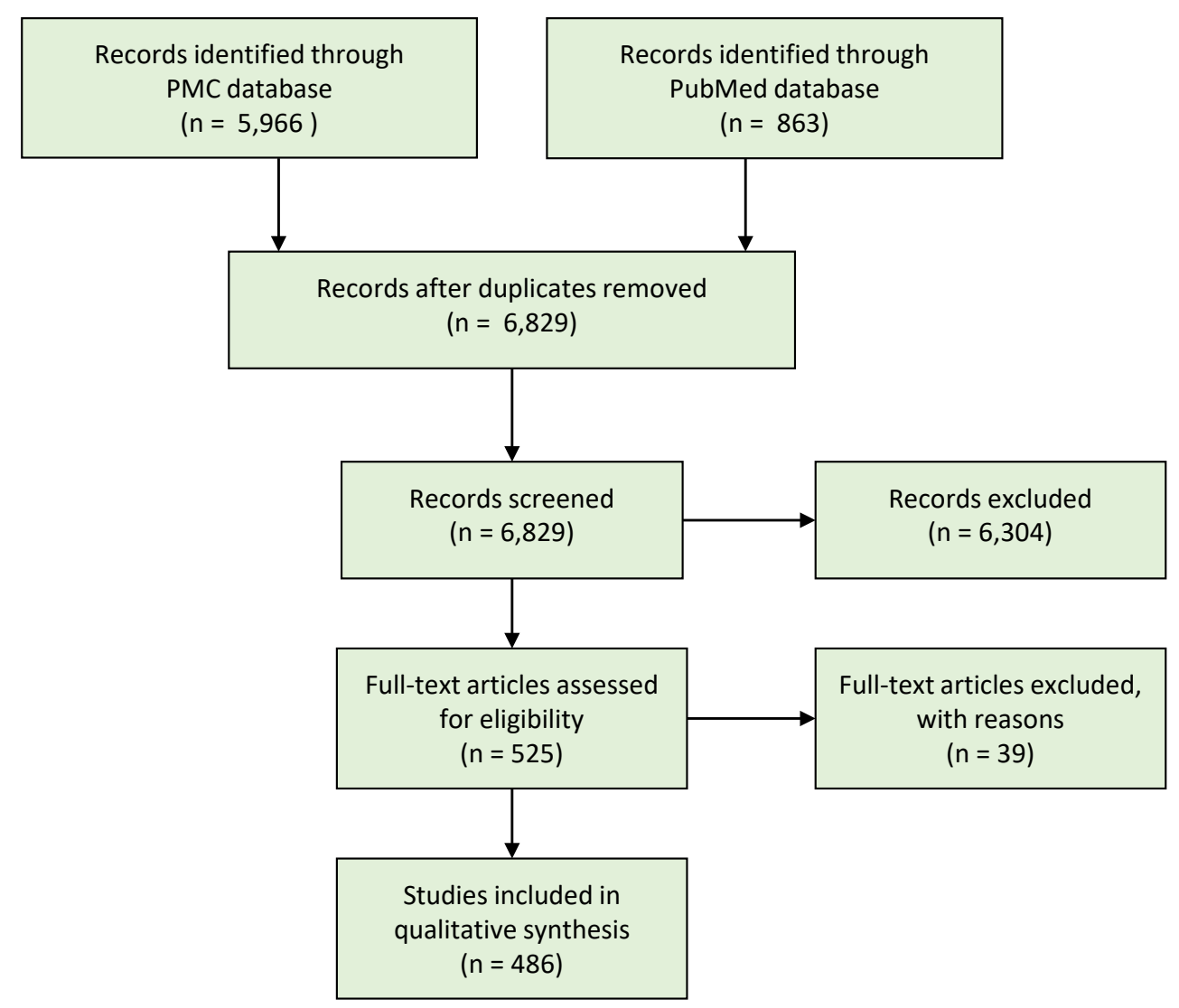

Figure 5: Systematic review procedure, following PRISMA reporting guidelines. 
medRxiv preprint doi: https://doi.org/10.1101/2020.10.30.20223529; this version posted November 4, 2020. The copyright holder for this preprint (which was not certified by peer review) is the author/funder, who has granted medRxiv a license to display the preprint in

It is made available under a CC-BY-NC-ND 4.0 International license .

\section{Supporting Information}

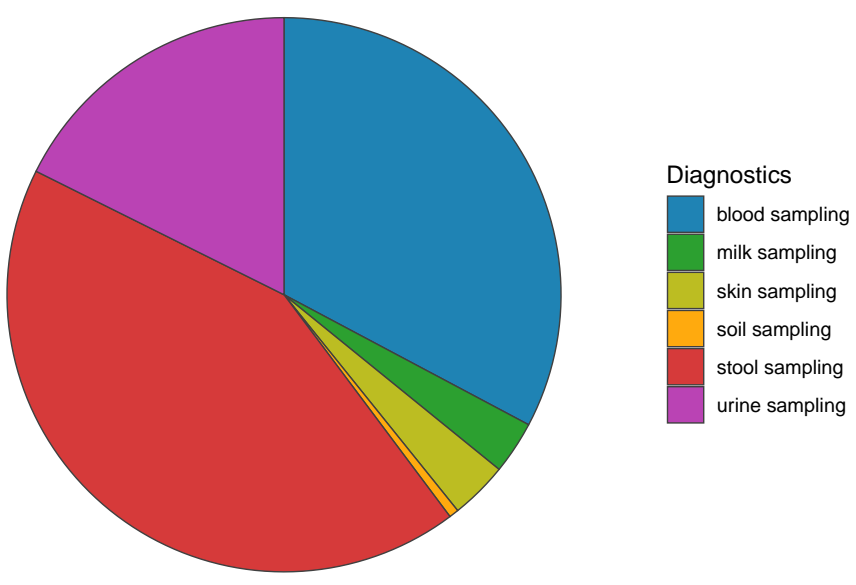

Supplementary Figure 1: Diagnostic method used to collect primary data in 357 studies that report new data from field surveillance. 


\section{References}

[1] Carlson CJ, Phillips AJ, Dallas TA, Alexander LW, Bansal S. What would it take to describe the global diversity of parasites? Proceedings of the Royal Society of London B. 2020;p. in press.

[2] GBD 2016 DALYs and HALE collaborators. Global, regional, and national disability-adjusted life-years (DALYs) for 333 diseases and injuries and healthy life expectancy (HALE) for 195 countries and territories, 1990-2016: a systematic analysis for the Global Burden of Disease Study 2016. The Lancet. 2017;390(10100):1260-1344.

[3] Pullan RL, Smith JL, Jasrasaria R, Brooker SJ. Global numbers of infection and disease burden of soil transmitted helminth infections in 2010. Parasites and Vectors. 2014;7(37).

[4] Weatherhead JE, Hotez PJ, Mejio R. The global state of helminth control and elimination in children. Pediatric Clinics of North America. 2017;64(4).

[5] Pabalan N, Singian E, Tabangay L, Jarjanazi H, Boivin MJ, Ezeamama AE. Soil-transmitted helminth infection, loss of education and cognitive impairment in school-aged children: A systematic review and meta-analysis. PLoS Neglected Tropical Diseases. 2018;12(1):e0005523.

[6] Mbabazi PS, Andan O, Fitzgerald DW, Chitsulo L, Engels D, Downs JA. Examining the relationship between urogenital schistosomiasis and HIV infection. PLoS Neglected Tropical Diseases. 2011;5(12):e1396.

[7] Carlson CJ, Mendenhall E, Singer S. Bringing syndemic theory into neglected tropical disease research. PLoS Neglected Tropical Diseases;p. in review.

[8] Dixon MA, Braae UC, Winskill P, Walker M, Devleesschauwer B, Gabriel S, et al. Strategies for tackling Taenia solium taeniosis/ cysticercosis: A systematic review and comparison of transmission models, including an assessment of the wider Taeniidae family transmission models. PLoS Neglected Tropical Diseases. 2019;13(4).

[9] Mostafa MH, Sheweita S, O'Connor PJ. Relationship between schistosomiasis and bladder cancer. Clinical Microbiology Reviews. 1999;12(1):97-111.

[10] Masaku J, Mutungi F, Gichuki PM, Okoyo C, Njomo DW, Njenga SM. High prevalence of helminths infection and associated risk factors among adults living in a rural setting, central Kenya: a cross-sectional study. Tropical Medicine and Health. 2017;45(15).

[11] GBD 2016 Disease and Injury Incidence and Prevalence Collaborators. Global, regional, and national incidence, prevalence, and years lived with disability for 328 diseases and injuries for 195 countries, 1990-2016: a systematic analysis for the Global Burden of Disease Study 2016. The Lancet. 2017;390(10100):1211-1259.

[12] World Health Organization. Helminth control in school[U+2010] age children: a guide for managers of control programmes. WHO Technical Report Series. 2011;2:1-90.

[13] World Health Organization. Ending the neglect to attain the sustainable development goals: A roadmap for neglected tropical diseases 2021-2030. 2020;.

[14] Nejsum P, Betson M, Bendall R, Thamsborg SM, Stothard J. Assessing the zoonotic potential of Ascaris suum and Trichuris suis: looking to the future from an analysis of the past. Journal of Helminthology. 2012;86(2):148-155. 
[15] Centers for Disease Control and Prevention. Dirofilariasis FAQs. 2012;

[16] Braae UC, Devleesschauwer B, Gabriël S, Dorny P, Speybroeck N, Magnussen P, et al. CystiSim-an agent-based model for Taenia solium transmission and control. PLoS Neglected Tropical Diseases. 2016;10(12).

[17] Salam N, Azam S. Prevalence and distribution of soil-transmitted helminth infections in India. BMC Public Health. 2017;17(201).

[18] Singer M, Bulled N, Ostrach B, Mendenhall E. Syndemics and the biosocial conception of health. The Lancet. 2017;389(10072):941-950.

[19] Raso G, Vounatsou P, Singer BH, N'Goran EK, Tanner M, Utzinger J. An integrated approach for risk profiling and spatial prediction of Schistosoma mansoni-hookworm coinfection. Proceedings of the National Academy of Sciences of the United States of America. 2006;103(18):6934-6939.

[20] Pullan RL, Bethony JM, Geiger SM, Cundill B, Correa-Oliveira R, Quinnell RJ, et al. Human Helminth Co-Infection: Analysis of Spatial Patterns and Risk Factors in a Brazilian Community. PLoS Neglected Tropical Diseases. 2008;2(12).

[21] Brooker S, Clements AC. Spatial heterogeneity of parasite co-infection: Determinants and geostatistical prediction at regional scales. International Journal for Parasitology. $2009 ; 39(5): 591-597$.

[22] Wanji S, Ndongmo WP, Fombad FF, Kengne-Ouafo JA, Njouendou AJ, Tchounkeu YF, et al. Impact of repeated annual community directed treatment with ivermectin on loiasis parasitological indicators in Cameroon: Implications for onchocerciasis and lymphatic filariasis elimination in areas co-endemic with Loa loa in Africa. PLoS Neglected Tropical Diseases. 2018;12(9).

[23] Adu-Gyasi D, Asante KP, Frempong MT, Gyasi DK, Iddrisu LF, Ankrah L, et al. Epidemiology of soil transmitted Helminth infections in the middle-belt of Ghana, Africa. Parasite Epidemiology and Control. 2018;3(3):e00071.

[24] Brooker SJ, Pullan RL, Gitonga CW, Ashton RA, Kolaczinski JH, Kabatereine NB, et al. Plasmodium-helminth coinfection and its sources of heterogeneity across east Africa. Journal of Infectious Diseases. 2012;205(5):841-852.

[25] Kabatereine NB, Standley CJ, Sousa-Figueiredo JC, Fleming FM, Stothard JR, Talisuna A, et al. Integrated prevalence mapping of schistosomiasis, soil-transmitted helminthiasis and malaria in lakeside and island communities in Lake Victoria, Uganda. Parasites and Vectors. 2011;4(1):232.

[26] Doumbo S, Tran TM, Sangala J, Li S, Doumtabe D, Kone Y, et al. Co-infection of long-term carriers of Plasmodium falciparum with Schistosoma haematobium enhances protection from febrile malaria: a prospective cohort study in Mali. PLoS Neglected Tropical Diseases. 2014;8(9):e3154.

[27] Stensgaard AS, Vounatsou P, Onapa AW, Simonsen PE, Pedersen EM, Rahbek C, et al. Bayesian geostatistical modelling of malaria and lymphatic filariasis infections in Uganda: predictors of risk and geographical patterns of co-endemicity. Malaria Journal. 2011;10(1):298. 
[28] Donahue RE, Cross ZK, Michael E. The extent, nature, and pathogenic consequences of helminth polyparasitism in humans: A meta-analysis. PLoS Neglected Tropical Diseases. $2019 ; 13(6)$.

[29] Adegnika AA, Kremsner PG. Epidemiology of malaria and helminth interaction: a review from 2001 to 2011. Current Opinion in HIV and AIDS. 2012;7(3).

[30] Standley CJ, Graeden E, Kerr J, Sorrell EM, Katz R. Decision support for evidence-based integration of disease control: A proof of concept for malaria and schistosomiasis. PLoS Neglected Tropical Diseases. 2018;12(4):e0006328.

[31] Mbah MLN, Skrip L, Greenhalgh S, Hotez P, Galvani AP. Impact of Schistosoma mansoni on malaria transmission in Sub-Saharan Africa. PLoS Neglected Tropical Diseases. 2014;8(10):e3234.

[32] Bergquist R, Elmorshedy H. Artemether and Praziquantel: Origin, Mode of Action, Impact, and Suggested Application for Effective Control of Human Schistosomiasis. Tropical Medicine and Infectious Disease. 2018;3(4).

[33] Derua YA, Kisinza WN, Simonsen PE. Differential effect of human ivermectin treatment on blood feeding Anopheles gambiae and Culex quinquefasciatus. Parasites and Vectors. 2015;8.

[34] Mekuriaw W, Balkew M, Messenger LA, Yewhalaw D, Woyessa A, Massebo F. The effect of ivermectin $(\mathrm{R}$ ) on fertility, fecundity and mortality of Anopheles arabiensis fed on treated men in Ethiopia. Malaria Journal. 2019;18(357).

[35] Slater HC, Walker PG, Bousema T, Okell LC, Ghani AC. The potential impact of adding ivermectin to a mass treatment intervention to reduce malaria transmission: a modelling study. The Journal of Infectious Diseases. 2014;210(12):1972-1980.

[36] Stensgaard AS, Vounatsou P, Onapa AW, Simonsen PE, Pedersen EM, Rahbek C, et al. Bayesian geostatistical modelling of malaria and lymphatic filariasis infections in Uganda: predictors of risk and geographical patterns of co-endemicity. Malaria Journal. 2011;10(298).

[37] Rajshekhar V. Neurocysticercosis: Diagnostic problems current therapeutic strategies. Indian Journal of Medical Research. 2016;144(3):319-326.

[38] Gripper LB, Welburn SC. The causal relationship between neurocysticercosis infection and the development of epilepsy - a systematic review. Infectious Diseases of Poverty. 2017;6(31).

[39] Centers for Disease Control and Prevention. Strongyloidiasis Epidemiology and Risk Factors. 2018;.

[40] World Health Organization. Strongyloidiasis Epidemiology;.

[41] Schär F, Trostdorf U, Giardina F, Khieu V, Muth S, Marti H, et al. Strongyloides stercoralis: global distribution and risk factors. PLoS Neglected Tropical Diseases. 2013;7(7).

[42] Borhani M, Fathi S, Lahmar S, Ahmed H, Abdulhameed MF, Fasihi Harandi M. Cystic echinococcosis in the Eastern Mediterranean region: Neglected and prevailing! PLOS Neglected Tropical Diseases. 2020;14(5):e0008114.

[43] Sadjjadi SM. Present situation of echinococcosis in the Middle East and Arabic North Africa. Parasitology International. 2006;55:S197-S202. 
[44] Centers for Disease Control and Prevention. Hymenolepiasis FAQs. 2012;.

[45] Cabada MM, Morales ML, Lopez M, Reynolds ST, Vilchez EC, Lescano AG, et al. Hymenolepis nana Impact among Children in the Highlands of Cusco, Peru: An Emerging Neglected Parasite Infection. The American Journal of Tropical Medicine and Hygiene. 2016;95(5):1031-1036.

[46] Vilchez Barreto PM, Gamboa R, Santivanez R, O’Neal SE, Muro C, Lescano AG, et al. Prevalence, Age Profile, and Associated Risk Factors for Hymenolepis nana Infection in a Large Population-Based Study in Northern Peru. The American Journal of Tropical Medicine and Hygiene. 2017;97(2):583-586.

[47] Magalhaes RJS, Fancony C, Gamboa D, Langa AJ, Sousa-Figueiredo JC, Clements AC, et al. Extending helminth control beyond STH and schistosomiasis: the case of human hymenolepiasis. PLoS Neglected Tropical Diseases. 2013;7(10):e2321.

[48] Cowie RH. Biology, Systematics, Life Cycle, and Distribution of Angiostrongylus cantonensis, the Cause of Rat Lungworm Disease. Hawai'i Journal of Medicine and Public Health. 2013;72(6 Suppl 2):6-9.

[49] Martins YC, Tanowitz HB, Kazacos KR. Central nervous system manifestations of Angiostrongylus cantonensis infection. Acta Tropica. 2014;141:46-53.

[50] Wen H, Vuitton L, Tuxun T, Li J, Vuitton DA, Zhang W, et al. Echinococcosis: Advances in the 21st Century. Clinical Microbiology Reviews. 2019;32(2).

[51] Deplazes P, Rinaldi L, Alvarez RC, Torgerson P, Harandi M, Romig T, et al. Global Distribution of Alveolar and Cystic Echinococcosis. Advances in Parasitology. 2017;95:315.

[52] Ta-Tang TH, Crainey JL, Post RJ, Luz SL, Rubio JM. Mansonellosis: current perspectives. Research and Reports in Tropical Medicine. 2018;9:9-24.

[53] May JM. Map of the world distribution of helminthiases. Geographical Review. 1952;42(1):98101.

[54] Molyneux D, Sankara DP. Guinea worm eradication: Progress and challenges - should we beware of the dog? PLoS Neglected Tropical Diseases. 2017;11(4):e0005495.

[55] Wilson-Aggarwal JK, Goodwin CE, Swan GJ, Fielding H, Tadesse Z, Getahun D, et al. Ecology of domestic dogs (Canis familiaris) as a host for Guinea worm (Dracunculus medinensis) infection in Ethiopia. Transboundary and Emerging Diseases. 2020;.

[56] Sripa B, Bethony JM, Sithithaworn P, Kaewkes S, Mairiang E, Loukas A, et al. Opisthorchiasis and Opisthorchis-associated cholangiocarcinoma in Thailand and Laos. Acta Tropica. 2010;120(Supplement 1):S158-S168.

[57] Banales JM, Cardinale V, Carpino G, Marzioni M, Andersen JB, Invernizzi P, et al. Expert consensus document: Cholangiocarcinoma: current knowledge and future perspectives consensus statement from the European Network for the Study of Cholangiocarcinoma (ENS-CCA). Nature Reviews Gastroenterology and Hepatology. 2016;13(5):261-280.

[58] Kim TS, Pak JH, Kim JB, Bahk YY. Clonorchis sinensis, an oriental liver fluke, as a human biological agent of cholangiocarcinoma: a brief review. BMB Reports. 2016;49(11):590-597. 
medRxiv preprint doi: https://doi.org/10.1101/2020.10.30.20223529; this version posted November 4, 2020. The copyright holder for this preprint (which was not certified by peer review) is the author/funder, who has granted medRxiv a license to display the preprint in It is made available under a CC-BY-NC-ND 4.0 International license .

[59] Li CD, Yang HL, Wang Y. Capillaria hepatica in China. World Journal of Gastroenterology. 2010;16(6):698-702.

[60] Saichua P, Nithikathkul C, Kaewpitoon N. Human intestinal capillariasis in Thailand. World Journal of Gastroenterology. 2008;14(4):506-510.

[61] Chai JY, Shin EH, Lee SH, Rim HJ. Foodborne Intestinal Flukes in Southeast Asia. The Korean Journal of Parasitology. 2009;47:S69-S102.

[62] Mas-Coma S, Bargues M, Valero M. Gastrodiscoidiasis, a plant-borne zoonotic disease caused by the intestinal amphistome fluke Gastrodiscoides hominis (Trematoda: Gastrodiscidae). Revista Ibérica de Parasitología. 2005;66:75-81.

[63] Dada-Adegbola H, Falade C, Oluwatoba O, Abiodun O. Gastrodiscoides hominis infection in a Nigerian-case report. West African Journal of Medicine. 2004;23(2):185-186.

796

[66] Dallas T. helminthR: an R interface to the London Natural History Museum's host-parasite database. Ecography. 2016;39(4):391-393.

64] Blum AJ, Hotez PJ. Global "worming": Climate change and its projected general impact on human helminth infections. PLoS Neglected Tropical Diseases. 2018;12(7).

[65] York EM, Butler CJ, Lord WD. Global decline in suitable habitat for Angiostrongylus (= Parastrongylus) cantonensis: the role of climate change. PLoS One. 2014;9(8):e103831.

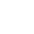

